\title{
Dopant activation mechanism of Bi wire- $\delta$-doping into Si crystal, investigated with wavelength dispersive fluorescence X-ray absorption fine structure and density functional theory
}

\author{
Koichi Murata, ${ }^{1,2, *}$ Christopher Kirkham, ${ }^{3,4}$ Masaru \\ Shimomura, ${ }^{5}$ Kiyofumi Nitta, ${ }^{6}$ Tomoya Uruga, ${ }^{6}$ Yasuko Terada, ${ }^{6}$ \\ Koh-ichi Nittoh, ${ }^{1}$ David R. Bowler, ${ }^{3,7}$ and Kazushi Miki ${ }^{1,2, \dagger}$ \\ ${ }^{1}$ National Institute for Materials Science (NIMS), \\ Namiki 1-1, Tsukuba, 305-0044, Japan \\ ${ }^{2}$ Faculty of Pure and Applied Sciences, University of Tsukuba, \\ Tennodai 1-1, Tsukuba, 305-8573, Japan ${ }^{\ddagger}$ \\ ${ }^{3}$ London Centre for Nanotechnology, University College London, \\ Gower Street, London WC1E 6BT, UK \\ ${ }^{4}$ Center for Computational Sciences, \\ University of Tsukuba, Tsukuba, 305-8577, Japan \\ ${ }^{5}$ Graduate School of Integrated Science and Technology, \\ Shizuoka University, Johoku 3-5-1, Nakaku, Hamamatsu, 432-8561, Japan \\ ${ }^{6}$ JASRI/ SPring-8, 1-1-1 Koto, Sayo-cho, Sayo-gun, Hyogo 679-5198, Japan \\ ${ }^{7}$ International Centre for Materials Nanoarchitectonics (MANA), \\ National Institute for Materials Science (NIMS) Namiki 1-1, Tsukuba, 305-0044, Japan
}

(Dated: February 2017; Revised ver20) 


\begin{abstract}
We successfully characterized the local structures of Bi atoms in a wire- $\delta$-doped layer $(1 / 8 \mathrm{ML})$ in a Si crystal, using wavelength dispersive fluorescence X-ray absorption fine structure at the beamline BL37XU, in SPring-8, with the help of density functional theory calculations. It was found that the burial of $\mathrm{Bi}$ nanolines on the $\mathrm{Si}(001)$ surface, via growth of $\mathrm{Si}$ capping layer at $400^{\circ} \mathrm{C}$ by molecular beam epitaxy, reduced the Bi-Si bond length from $2.79 \pm 0.01 \AA$ to $2.63 \pm$ $0.02 \AA$. We infer that following epitaxial growth the Bi-Bi dimers of the nanoline are broken, and the $\mathrm{Bi}$ atoms are located at substitutional sites within the Si crystal, leading to the shorter Bi-Si bond lengths.
\end{abstract}

\footnotetext{
*kmurata@g.ucla.edu

† MIKI.Kazushi@nims.go.jp

$¥$ Permanent address: Central Research Institute of Electric Power Industry, 2-6-1 Nagasaka, Yokosuka, Kanagawa, 240-0196, Japan
} 
Recently, it has been proposed and demonstrated that heavy elements are potentially suitable for new computing schemes using bound electrons for high temperature operation, e.g. quantum information processing [1-4]. However, in practice doping heavy elements into the Si crystal is difficult for a variety of reasons. These include, their low diffusion coefficient and solid solubility for thermal diffusion methods [5], their strong tendency towards surface segregation during molecular beam epitaxy (MBE) [6], and accompanied defect formation during ion implantation $[7,8]$.

To this end, we proposed a new solution to realize heavy element doping, through the use of their nanostructures on the Si surface. One demonstration was highly concentrated Bi doping into a Si crystal using Bi nanolines as a dopant source [9]. This doping process consists of two steps. Firstly, burial of Bi nanolines in an epitaxial Si layer [10]. Secondly, activation of the dopants by annealing at $1200{ }^{\circ} \mathrm{C}$ [11]. Activation was confirmed by photoluminescence. Successful doping requires that Bi atoms are located at substitutional lattice sites, with four-fold Si coordination, and that defects (i.e. optical recombination centers) are eliminated. The necessity of high temperature annealing means that at least one of these two features is missing after the initial burial.

The Bi nanoline consists of two parallel lines of Bi dimers, which run perpendicular to the Si dimer rows of $\mathrm{Si}(001)$ [10], with a width equivalent to four Si dimers (15.4 $\AA$ ). Based on experimental results and comprehensive DFT calculations, the Haiku model of the Bi nanoline was proposed [12]. The Haiku model consists of two parallel lines of Bi dimers sitting above a large reconstructed Si region, known as the Haiku core as show in Fig. 1 [13]. The Haiku model was recently verified by experiment, where a hydrogen cracker was used to remove the $\mathrm{Bi}$ from the Bi nanoline, whilst leaving the Haiku core intact, allowing for its direct observation [14].

In this work we will clarify the physical processes which take place during burial of $\mathrm{Bi}$ nanolines in an epitaxial Si layer, through the use of two techniques for analyzing our dopant samples, X-ray absorption fine structure (XAFS) and Density Functional Theory (DFT). For our analyses we prepared two samples, with the Bi nanoline buried by a Si capping layer under different conditions. One grown at a temperature of $400{ }^{\circ} \mathrm{C}$ with a $1 \mathrm{ML}$ Bi surfactant, and the other at room temperature. It is difficult to analyze the structure of buried Bi nanolines, due to the relatively dilute Bi in the sample $\left(1 / 8 \mathrm{ML}, 8.48 \times 10^{13} \mathrm{~cm}^{-2}\right)[15]$. Therefore we employed the wavelength dispersive fluorescence X-ray absorption fine struc- 


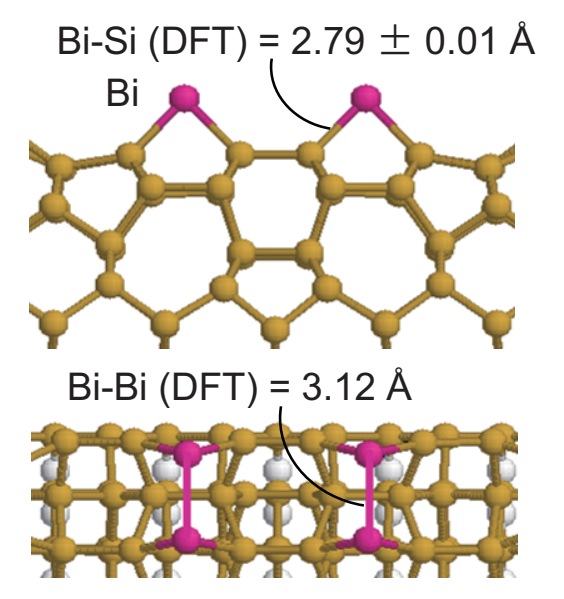

FIG. 1. Haiku model of the Bi nanoline [13] shown from the side and above. Bi atoms are shown in pink, $\mathrm{Si}$ in brown and $\mathrm{H}$ in white.

ture (XAFS) technique [16] at the beamline BL37XU, with an undulator, in a synchrotron radiation facility, SPring-8. This technique is a powerful tool to extract XAFS signals of very dilute elements from the intense background scattering and fluorescence X-rays of the bulk sample.

We used an ion-pumped Si MBE system for Si sample growth, and a resistively-heated effusion cell for the evaporation of $\mathrm{Bi}$, following the procedures from our previous work [11]. The base pressure was $2 \times 10^{-7} \mathrm{~Pa}$. The growth rate of $\mathrm{Si}$ was $0.3 \mathrm{ML} \mathrm{s}^{-1}(1 \mathrm{ML}=$ $6.8 \times 10^{14}$ atoms $\mathrm{cm}^{-2}$ ). A $20 \mathrm{keV}$ reflection high-energy electron diffraction (RHEED) system was used for monitoring the surface structure of Si. Si substrates were subjected to the standard cleaning process and then immediately loaded into the growth chamber. After removal of the protective oxide, an atomically clean surface with a sharp $2 \times 1$ RHEED pattern was established by depositing a $100 \mathrm{~nm}$ thick Si buffer layer at $400{ }^{\circ} \mathrm{C}$ followed by thermal annealing at $800{ }^{\circ} \mathrm{C}$. $\mathrm{Si}(001)$ substrates, whose surface normals were miscut at $4^{\circ}$ off towards [110] from (001), were used to make single domain $\mathrm{Si}(001)$ surfaces for the fabrication

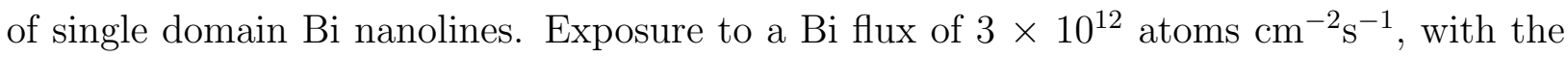
substrate maintained at around $600{ }^{\circ} \mathrm{C}$, resulted in characteristic one-dimensional RHEED patterns, i.e. a clear signature of the Bi nanolines. After formation of the Bi nanolines, a $50 \mathrm{~nm}$-thick Si capping layer was epitaxially grown with a $1 \mathrm{ML}$ Bi surfactant layer at the growth temperature $T_{\text {sub }}=400{ }^{\circ} \mathrm{C}$ [15]. The Bi surfactant formed a surface segregation layer, which was removed by chemical etching [17]. For the other sample, 30 nm-thick of 
amorphous Si was deposited at room temperature. As a reference sample, a $\mathrm{Si}(001)$ wafer implanted with $2.31 \times 10^{14} \mathrm{~cm}^{-2} \mathrm{Bi}^{+}$ions ( $\left.\mathrm{Si}: \mathrm{Bi}\right)$ was prepared, and annealed at $600{ }^{\circ} \mathrm{C}$ for 30 min in $\mathrm{N}_{2}$, following a recipe from the literature [7]. Metal Bi crystal powder diluted in BN powder was used as a standard sample for the XAFS experiments.

XAFS measurements were performed at the Bi $\mathrm{L}_{I I I^{-}}$-edge in $\mathrm{N}_{2}$ at the beamline BL37XU, in SPring-8. The X-rays from the undulator source were monochromatized by a $\operatorname{Si}(111)$ double-crystal monochromator. Higher harmonics of the incident X-rays were removed by Rh coated double mirrors. The incident X-ray intensity was measured by an ionization chamber filled with a 70/30 \% He/N gas mixture. The XAFS spectra were measured in the wavelength dispersive fluorescence mode using a 19-element Ge detector with a bent crystal Laue analyzer (DCA-0950, Oxford Danfysik), placed in front of the Ge detector. This was done to remove the intense scattered X-rays from the sample and enhance the signal to background ratio in the fluorescence XAFS spectra. The sample was mounted on a swivel stage to set the incident angle between the X-rays and the sample surface at $4^{\circ}$. The polarization of the incident X-rays was parallel to the sample surface (Fig. 2 (a)). The XAFS spectra were analyzed using the Demeter software [18], after removing diffraction noise. Theoretical scattering phase shifts and amplitudes were calculated using FEFF8 [19]. Curve fitting was performed to determine the distances $(\mathrm{R})$, the coordination numbers $\left(\mathrm{CN}^{*}\right)$, the Debye-Waller factors $\left(\sigma^{2}\right)$, and the relative error between the fit and data (R-factor) for each sample, with an energy shift parameter $\Delta E$ used to align the theoretical spectrum to the measured spectrum. In most cases, diffraction noise arising from the single crystal Si substrate was removed in the XAFS spectra by the continuous rotation of the sample around the surface normal. Since polarized X-rays were used, the $\mathrm{CN}^{*}$ has a polarization dependence $\left(\sim 1 / 2 \sum\left(1+3 \cos ^{2} \theta\right)\right.$ [20]) for anisotropic specimens. Here, $\theta$ is the angle between the polarization of the X-ray and sample bonds. Intense diffraction noise was only observed for the case of incident X-rays parallel to the Bi nanoline axis (data not shown here), indicating that the one dimensional nature of the Bi nanoline was maintained after burial. This is consistent with reciprocal X-ray imaging results. Since the typical length of Bi nanolines is over $500 \mathrm{~nm}$ [10], Bragg's diffraction condition would be satisfied.

Theoretical calculations were used to study two different systems, Si adsorption on the Bi nanoline, and Bi in bulk Si. Calculations were performed using Density Functional Theory (DFT) [21, 22], as implemented in the Vienna Ab initio Simulation Package (VASP) [23, 24], 


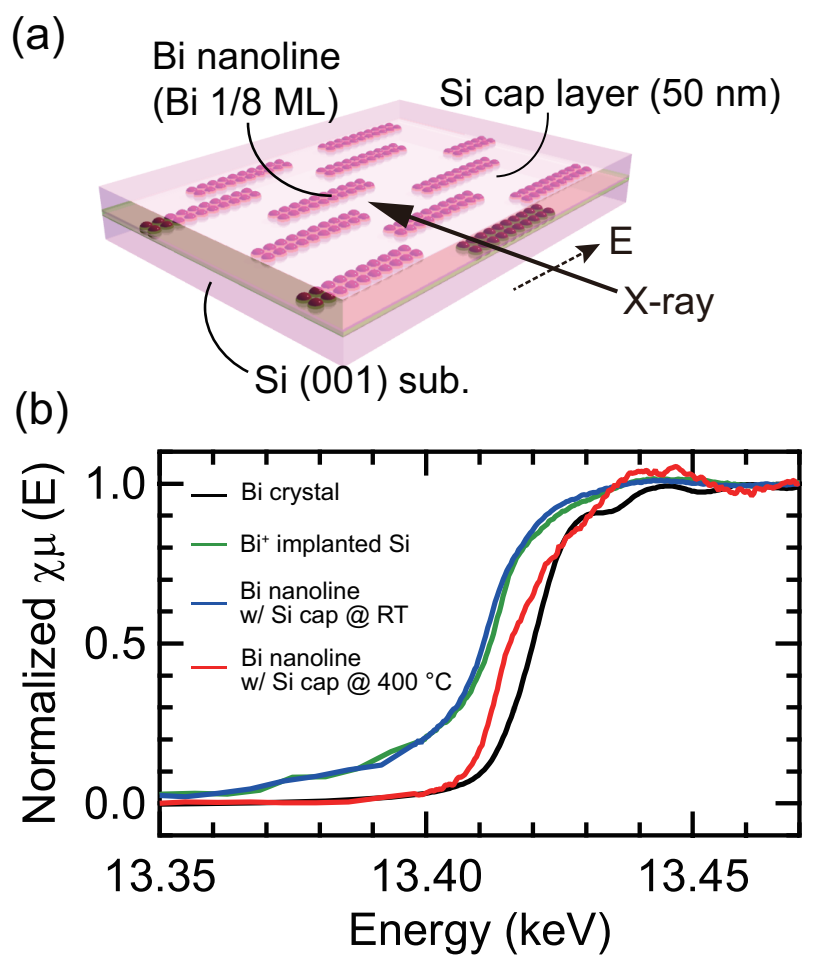

FIG. 2. (a) Schematic illustration of the sample structure and geometry for XAFS measurements. (b) Comparison of $\mathrm{Bi} \mathrm{L}_{I I I}$-edge XANES spectra of our two samples, buried Bi nanolines with a $\mathrm{Si}$ capping layer grown at $\mathrm{RT}$ or $400{ }^{\circ} \mathrm{C}$ (w/ Bi surfactant), a reference $\mathrm{Bi}^{+}$implanted sample, and a standard Bi metal powder sample.

with slightly different settings for each system.

For Bi nanoline calculations the PW91 exchange-correlation functional [25] was used, whereas for bulk calculations the gradient-corrected Perdew-Burke-Ernzerhof (PBE) [26] exchange-correlation functional was used. For Bi the $6 s^{2} 6 p^{3}$ electrons were treated as valence, the rest as core. The core electrons were described by the projector augmented wave (PAW) method [27, 28].

The Bi nanoline and the surrounding $\operatorname{Si}(001)$ surface were represented by a ten layer slab model, with the $\mathrm{p}(2 \times 2)$ surface reconstruction, using the experimental Si bulk lattice constant of $a_{o}=5.4306 \AA$. The tenth layer was terminated by $\mathrm{H}$ atoms in a dihydride structure, and both the $\mathrm{H}$ atoms and the bottom two Si layers were fixed. The surface was represented by two Si dimers rows, each equivalent to ten dimers long, consisting of six regular dimers and the Haiku reconstructed region spanning the remaining four. The Bi 
(a) Substitutional Bi

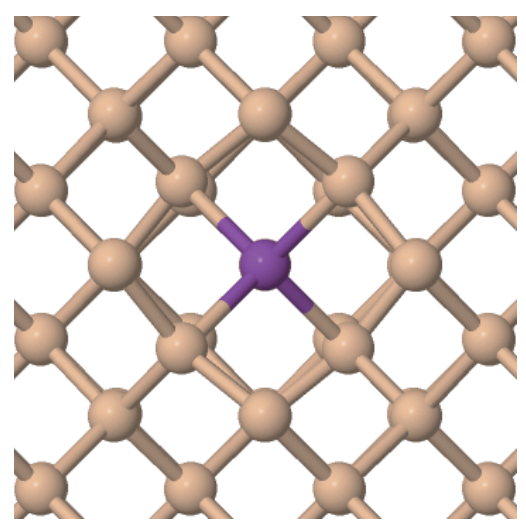

(b) Substitutional Bi with vacancy $(\mathrm{Bi}-\mathrm{V})$

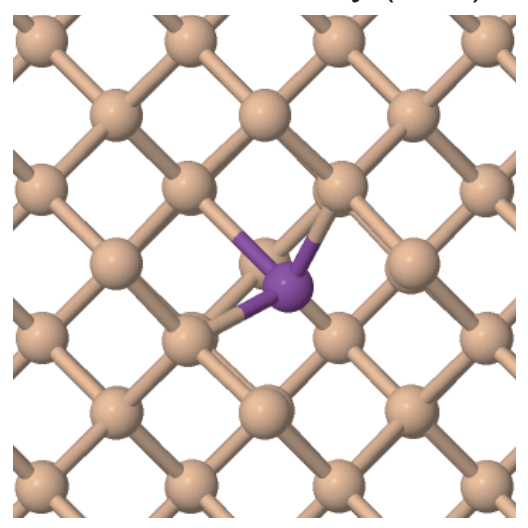

(c) Two substitutional Bi

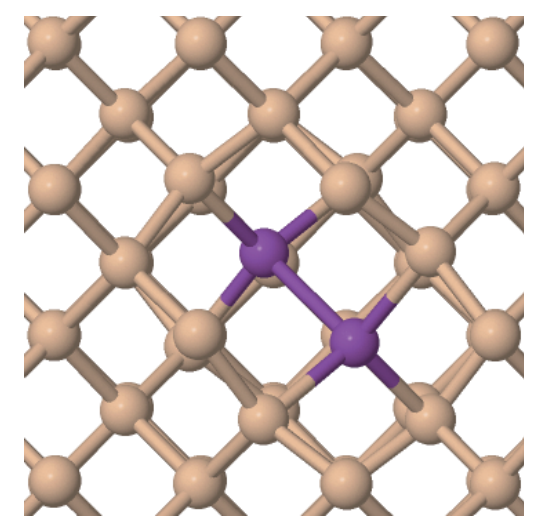

FIG. 3. Structural models of Bi in Si. (a) Substitutional Bi, (b) substitutional Bi and an Si vacancy and (c) two substitutional Bi.

nanoline itself consists of a pair of Bi dimers per dimer row, sitting atop the Haiku region. To model adsorption a single Si atom was placed above a Bi dimer and the structure allowed to relax. Periodic images were separated by a vacuum gap of $12.73 \AA$ to prevent interactions.

A 512 atom bulk Si cell was used to study Bi defects, using the calculated theoretical lattice constant of $5.469 \AA$. It has previously been shown that a cell of this size is required for defect calculations [29]. Cells containing between zero and two Bi atoms were considered. For cells containing no Bi, the simplest mono- and di-vacancies [29, 30] were considered. Single Bi atoms were then inserted at one of the vacanct lattice sites to create substitutional $\mathrm{Bi}\left(\mathrm{Bi}_{\text {subst }}\right)$ and $\mathrm{Bi}$ with vacancy $(\mathrm{Bi}-\mathrm{V})$ structures. For cells containing two Bi defects, we considered from the nearest to next next nearest neighbour sites. Structural models for several of these Bi defects are shown in Fig. 3.

Bi nanoline calculations used an energy cut-off of $250 \mathrm{eV}$ with a $2 \times 1 \times 1$ MonkhorstPack k-mesh along the direction of the Bi nanoline. Bulk calculations used an energy cut-off of $300 \mathrm{eV}$ with a $2 \times 2 \times 2 \mathrm{k}$-point mesh. All calculations were spin polarised with no restrictions placed on the spins. In both cases geometry optimisations were performed with a $0.02 \mathrm{eV} / \AA$ convergence condition for the forces on each atom.

For the Bi nanoline, adsorption energy was calculated using

$$
E_{a d s}=E_{n a n o+S i}-E_{n a n o}-E_{S i}
$$

where $E_{\text {nano }+S i}$ is the energy of the structure with $\mathrm{Si}$ adsorbed, $E_{\text {nano }}$ is the energy of the 


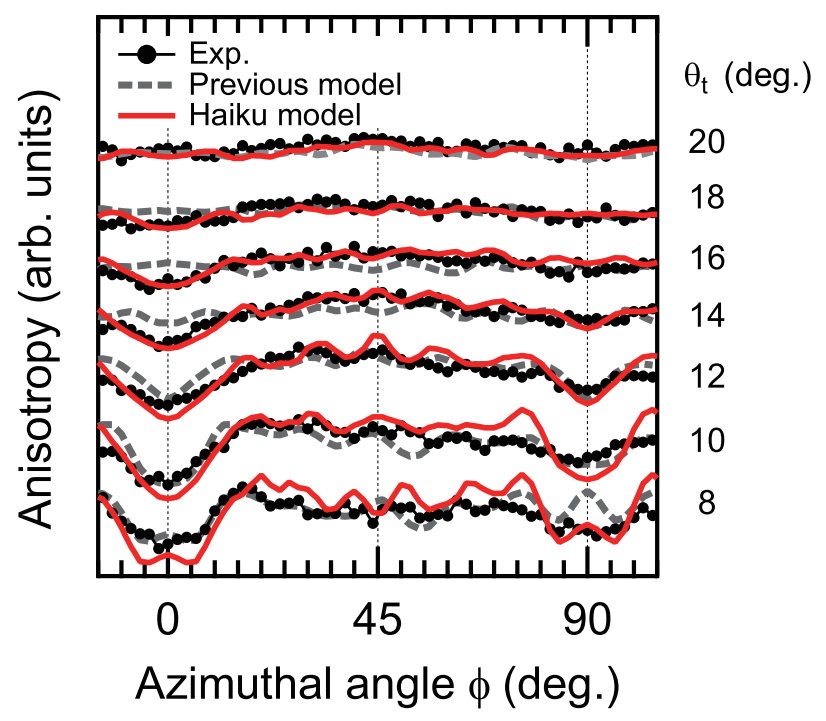

FIG. 4. Bi $4 \mathrm{~d}_{5 / 2}$ azimuthal XPD patterns of Bi nanoline on $\mathrm{Si}(001)$ for $\theta_{t}=8-20$. Solid circles connected with thin lines are experimental and gray dashed lines are the results of simulations for the previous model [31]. The red solid lines are the result of simulation for the Haiku model.

Bi nanoline and $E_{S i}$ is the energy of an atomic Si.

For bulk defects two energy measures were considered. For single Bi defects we calculated the stability of the defect structures relative to a vacancy structure, according to

$$
\Delta E=E_{S i+B i}-E_{S i+V}-E_{B i},
$$

where $E_{S i+B i}$ is the energy of the bulk cell containing a Bi defect, $E_{S i+V}$ is the energy of bulk cell with one or two vacancies and $E_{B i}$ is the energy of an atomic Bi. For double Bi defects we calculated their energies relative to the most stable configuration.

We provided further validation of the Haiku model by reconsidering the X-ray photoelectron diffraction (XPD) patterns as shown in Fig. 4, where the simulated anisotropy pattern of the Haiku model is plotted against experimental data and the older Miki model [31]. These XPD patterns were calculated using a multiple scattering custer (MSC) framework [32] which is more accurate for the linearly arranged system. The Haiku model shows a better agreement with experiment than the Miki model, especially around the azimuthal angles of 0 and 90 degrees, the former of which corresponds to the Bi dimer bond direction. Thus, structural parameters obtained by DFT are also acceptable by XPD. From the Haiku model, the Bi-Si bond length is calculated to be $2.79 \pm 0.01 \AA$, so will be used in the following 

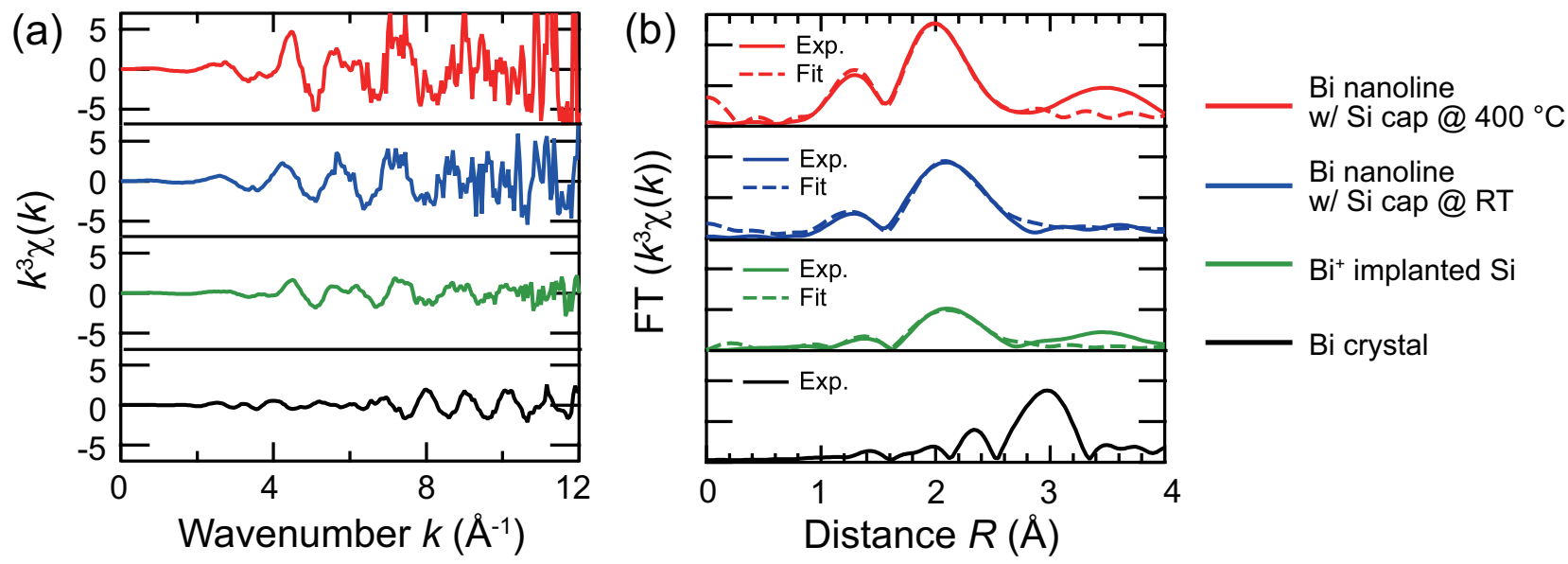

FIG. 5. (a) Bi L LII-edge $k^{3}$-weighted EXAFS spectra of our two samples, buried Bi nanolines with a Si capping layer grown at $\mathrm{RT}$ or $400{ }^{\circ} \mathrm{C}$ (w/ Bi surfactant), a reference $\mathrm{Bi}^{+}$implanted sample, and a standard Bi metal powder sample. (b) Their Fourier transform at $k=2-10 \AA^{-1}$, with their curves fitted by a one-shell model using the parameters from Table I.

TABLE I. Structural parameters of the Bi-Si nearest neighbour shells obtained from the curve fitting analysis of the Bi $\mathrm{L}_{I I I}$-edge EXAFS spectra of our two samples, buried Bi nanolines with a Si capping layer grown at $\mathrm{RT}$ or $400{ }^{\circ} \mathrm{C}$ (w/ Bi surfactant), a reference $\mathrm{Bi}^{+}$implanted sample, and a standard Bi metal powder sample. $2<k<10 \AA^{-1}, 1.6<r<2.7 \AA$. For bulk Bi, structural parameters of the Bi-Bi nearest neighbour shell are shown.

\begin{tabular}{lccccc}
\hline \hline Samples & $\mathrm{R}(\AA)$ & $C N^{*}$ & $\sigma^{2}\left(\AA^{2}\right)$ & $\Delta E(\mathrm{eV})$ & $\mathrm{R}$-factor \\
\hline (A) $\mathrm{Si}$ cap at $400{ }^{\circ} \mathrm{C}$ & $2.63 \pm 0.02$ & $4.4 \pm 0.5$ & $0.0081 \pm 0.0021$ & -1.49 & 0.00084 \\
(B) $\mathrm{Si}$ cap at $\mathrm{RT}$ & $2.670 \pm 0.003$ & $0.9 \pm 0.5$ & $0.0041 \pm 0.0003$ & -1.67 & 0.00007 \\
$(\mathrm{C}) \mathrm{Bi}^{+}$ion implanted & $2.63 \pm 0.01$ & $1.1 \pm 0.5$ & $0.003 \pm 0.001$ & 0.47 & 0.00033 \\
(D) $\mathrm{Bi}$ bulk (Bi-Bi bond) & $3.075 \pm 0.004$ & $2.3 \pm 0.5$ & $0.0055 \pm 0.0012$ & - & 0.02918 \\
\hline \hline
\end{tabular}

discussions.

Figure 5 shows the Bi $\mathrm{L}_{I I I^{-}}$-edge extended XAFS (EXAFS) spectra and the results of the curve fitting as listed in Table I. The curve fitting was made using a one-shell model with Bi-Si scattering paths. It should be noted that the $x$-axis in Fig. $5(\mathrm{~b})$ does not show the correct distance $R$, because these Fourier transforms did not take into account the phase shift in the photo-electron scattering process. Thus the values from curve fitting in Table I 
(a)

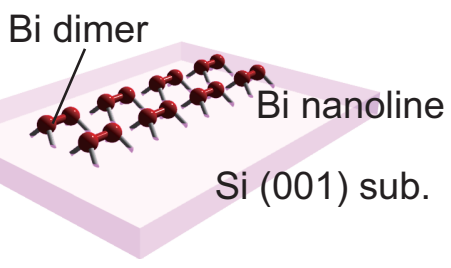

(b) i. original

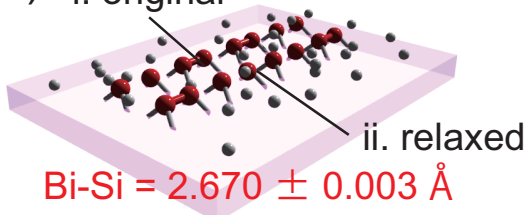

(c)

$\mathrm{Bi}-\mathrm{Si}=2.63 \pm 0.02 \AA$
Si cap layer

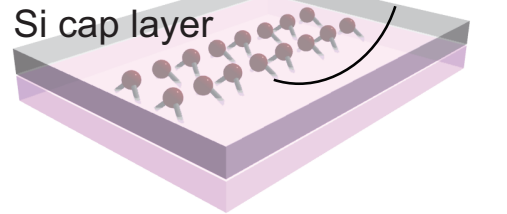

FIG. 6. Schematic illustration of the burial of Bi nanolines into a Si crystal. (a) Bi nanolines on $\mathrm{Si}(001),(\mathrm{b}) \mathrm{w} / \mathrm{cap} @ \mathrm{RT},(\mathrm{c}) \mathrm{w} /$ cap $@ 400{ }^{\circ} \mathrm{C}$.

are correct. In the Fourier transform (FT)-EXAFS spectra of the buried Bi nanolines and in $\mathrm{Bi}^{+}$implanted Si (Fig. 5(b)), no peaks from Bi-Bi scattering path were observed. However, we could not rule out the existence of Bi dimer structures in these samples based on EXAFS observations. The quality of the $\chi(k)$ EXAFS spectra is not high enough for precise curve fitting in the region of $k>8 \AA^{-1}$ in Fig. 5(a), where the contribution of the Bi-Bi scattering path to the EXAFS signal is significant. Also, the Debye-Waller factor of the Bi-Bi bond is large even in the Bi metal, which indicates the EXAFS amplitude of the Bi-Bi scattering path is quite small in general for Bi complexes. Although we cannot definitively rule out the existence of Bi-Bi bonding in our samples, our lack of evidence means we cannot provide a measure of the Bi-Bi bond length, and will hereafter only discuss Bi-Si bonding. As shown in Table I, the Bi-Si bond length is $2.63 \pm 0.02 \AA$ for the Bi nanolines capped at $400{ }^{\circ} \mathrm{C}$. Remarkably this $\mathrm{Bi}-\mathrm{Si}$ bond length is the same as the $\mathrm{Bi}^{+}$ion implanted $\mathrm{Si}$ although the $C N^{*}$ is different. The $C N^{*}$ of $\mathrm{Bi}-\mathrm{Si}$ is $4.4 \pm 0.5$ for the $\mathrm{Bi}$ nanolines capped at $400{ }^{\circ} \mathrm{C}$, which is close to the ideal value for substitutional $\mathrm{Bi}$ in a $\mathrm{Si}$ crystal.

As specified earlier, DFT calculations were employed to gain more insight into this system. Based on eq. 2, $\Delta E$ for $\mathrm{Bi}_{\text {subst }}$ and $\mathrm{Bi}-\mathrm{V}$ are -4.611 and $-4.630 \mathrm{eV}$, respectively. It is marginally more favorable for Bi to fill a di-vacancy than a mono-vacancy, but the di-vacancy is likely more unstable in the first place. For two nearby Bi defects we found that the most distant configuration, at next next nearest neigbour sites was the most stable, meaning that $\mathrm{Bi}$ atoms prefer to be separated. However, the relationship between Bi-Bi distance and energy is not straightforward, since $\mathrm{Bi}$ at nearest sites $(+0.044 \mathrm{eV})$ were found to be more stable than at next nearest sites $(+0.107 \mathrm{eV})$. This difference can likely be accounted for 
by the fact that a Bi-Bi bond has been replaced with a Bi-Si bond. It has been suggested that $\mathrm{Bi}$ in Si may display negative effective $\mathrm{U}$ behavior [33, 34], which can be assessed by considering the relative energetics of different charge states. However, we could find no evidence for this behavior for any of our defect structures.

The Bi-Si bond lengths for $\mathrm{Bi}_{\text {subst }}$ and $\mathrm{Bi}-\mathrm{V}$ were calculated to be 2.65 and $2.78 \AA$, respectively. A vacancy allows for the release of compressive strain, as evidenced by the lower energy, elongating the bond length. However, based on the Bi-Si bond length, the $\mathrm{Bi}-\mathrm{V}$ structure cannot be fitted to the experimental results. When two Bi were considered, the Bi-Si bond lengths were always found to be $2.65 \AA$. Thus, even though separated Bi are energetically favoured, structural parameters do not change with distance. Hence, both single and double substitutional $\mathrm{Bi}$ are promising structures for embedded Bi.

In Fig. 2 (b), the X-ray absorption $\mathrm{Bi}_{I I I}$-edge of $\mathrm{Bi}$ atoms in $\mathrm{Si}$ is shifted to lower energy than that of the Bi crystal. Unexpectedly, the absorption edge of the Bi ion implanted sample is located at the lowest energy position. This indicates that a majority of the $\mathrm{Bi}$ atoms are associated with defects, such as vacancies (V), and/or form Bi clusters in the Si crystal. It should be noted that $\mathrm{Bi}-\mathrm{V}$ structures are observed on the $\mathrm{Si}(001)$ surface of $\mathrm{Bi}$ ion implanted Si after high temperature anneal at $1100{ }^{\circ} \mathrm{C}$ [35], although this depends on the implantation dose and post-annealing. However, DFT calculations indicate that Bi-V structures show an increased Bi-Si bond length $(2.78 \AA)$, in contradiction to the observations for this sample. Taken together with the fact that the current sample is highly dosed, it appears more likely that $\mathrm{Bi}$ clusters are present in the ion implanted samples. This would also explain why the $C N^{*}$ of the ion implanted sample is quite low $(1.1 \pm 0.5)$, since Bi clusters would reduce the number of Bi-Si bonds. By comparison, the absorption edge for buried Bi nanolines is located at a higher energy position, suggesting that the Bi atoms are at substitutional sites. Preliminary DFT calculations support the observed absorption edge shifts, and the presence of Bi clusters. A more detailed analysis will be forthcoming.

To further discuss the physical mechanism for burial of Bi nanolines into the Si crystal, the difference of $\mathrm{Bi}$-Si bond lengths before and after $\mathrm{Si}$ capping were compared and two features noted. Firstly, the Bi-Si bond length in the epitaxial Si layer $(2.63 \AA)$ is shorter than that of Bi nanolines on $\mathrm{Si}(001)$. Secondly, the Bi-Si bond length for amorphous cap growth at RT $(2.67 \AA)$ is longer than for epitaxial cap growth $(2.63 \AA)$. Focusing on the second point, according to previous X-ray standing wave (XSW) experiments for RT growth, the 
Bi nanoline structure was found to persist beneath an amorphous capping layer [36]. This is also consistent with the reciprocal X-ray imaging results (See Supplementary Information). Therefore, if we assume that the nanoline structure at least partly survives RT growth, the low $C N^{*}$ can be explained by the X-ray polarization dependence of an anisotropic structure. For the present measurement geometry (the polarization of the X-ray is parallel to the Bi-Bi bond), the angle $\theta$ between the polarization of the X-ray and the two Bi-Si bonds with the subsurface is almost $90^{\circ}$. Thus the $\mathrm{CN}^{*}$ is minimized.

In an attempt to tackle the discrepancy in bond lengths, we considered Si deposition onto the Bi nanoline. We calculated a single Si adatom adsorbed at the centre of a Bi dimer. From eq. 1 the adsorption energy $\left(E_{a d s}\right)$ was calculated to be $-1.302 \mathrm{eV}$. This is relatively low, but shows that adsorption of $\mathrm{Si}$ on the nanoline is possible. The adsorbed Si forms two Bi-Si bonds, both of $2.69 \AA$, close to that observed for an amorphous Si cap. In addition, the surface Bi-Si bonds change from $2.79 \pm 0.01 \AA$ to $2.84 \pm 0.01 \AA$. However, when all six bonds are averaged, the value is the same as the clean case, $2.79 \AA$. Si adsorption alone cannot explain the amorphous capping situation.

We instead propose that for amorphous Si capping, partial nanoline reconstruction occurs as shown in Fig.6(b). We assume that some Bi are at relaxed substitutional sites (Bi-Si bond length $2.63 \AA$ ), whilst others remain in the original nanoline arrangement, or with $\mathrm{Si}$ adsorbed (Bi-Si bond length $2.79 \AA$ ). Under this assumption, if roughly $25 \%$ of the nanolines remained in their original structure, then the averaged bond length would match to that observed in experiment $(2.67 \AA)$. The relaxed to unrelaxed ratio would significantly depend on the temperature of the substrate during the growth process. By assuming full relaxation during $1 \mathrm{ML}$ Si growth at $400{ }^{\circ} \mathrm{C}$, since the bond lengths match to the ion implanted sample, the calculated binding energy of $\mathrm{Bi}$ atoms in the $\mathrm{Bi}$ nanoline is less than $1.9 \mathrm{eV}$ (See Supplementary Information for full details). In other words, our results suggest that Bi nanolines partially survive burial at room temperature, whereas at $400{ }^{\circ} \mathrm{C}$ the structure is fully relaxed to substitutional positions within the Si crystal.

In conclusion, the characterization of the local structures of $\mathrm{Bi}$ atoms in a wire- $\delta$-doped layer $(1 / 8 \mathrm{ML})$ of a $\mathrm{Si}$ crystal was achieved with wavelength dispersive fluorescence X-ray absorption fine structure using a bent crystal Laue-type analyzer, and the support of DFT calculations. We provide analysis for several different sample preparation conditions, including ion implantation, and Bi nanolines buried beneath epitaxially grown and amorphous Si 
caps. Although each scenario is likely complicated, containing a mixture of different Bi structures, we propose the most significant arrangement for each. Ion implanted samples contain substitutional Bi, but also defects such as vacancies or more likely Bi clusters, due to the implantation process. Burial of the Bi nanolines with an epitaxially grown Si capping layer at $400{ }^{\circ} \mathrm{C}$ reduces the Bi-Si bond lengths from $2.79 \pm 0.01 \AA$ on the surface, to 2.63 $\pm 0.02 \AA$. The same Bi-Si bond length is observed for Bi ion implanted samples, so we infer that the end structures are the same, except that the buried Bi nanoline lacks the defects usually associated with ion implantation. Both are in good agreement with the calculated bond length for $\mathrm{Bi}$ at substitutional sites within bulk Si, making these suitable for doping states in a Si crystal. During room temperature deposition of an amorphous capping layer, we suppose that the $\mathrm{Bi}$ nanolines are partially relaxed, with a fraction surviving the capping process. Adsorption of $\mathrm{Si}$ on the nanoline is suggested as a potential starting point for burial.

The synchrotron radiation experiments were performed at the beamline BL37XU, SPring8 with the approval of the Japan Synchrotron Radiation Research Institute (JASRI) (Proposal Nos. 2011B1692, 2012A1546, and 2012B1664). Calculations were performed using the UCL Legion High Performance Computing Facility, and associated support services. We acknowledge Dr. Sakata at NIMS for technical help. This research was supported by JSPS KAKENHI Grant Numbers 20246017 (K. Miki) and 20121355 (K. Murata), 26105007 (M. S.), and also NIMS Nanofabrication Platform in Nanotechnology Platform Project from the Ministry of Education, Culture, Sports, Science and Technology, Japan (MEXT).

[1] A. M. Stoneham, A. J. Fisher, and P. T. Greenland, J. Phys. Condens. Matter 15, L447 (2003).

[2] G. W. Morley, M. Warner, A. M. Stoneham, P. T. Greenland, J. v. Tol, C. W. M. Kay, and G. Aeppli, Nat. Mater. 9, 725 (2010).

[3] J. J. L. Morton, A. M. Tyryshkin, R. M. Brown, S. Shankar, B. W. Lovett, A. Ardavan, T. Schenkel, E. E. Haller, J. W. Ager, and S. A. Lyon, Nature 455, 1085 (2008).

[4] R. E. George, W. Witzel, H. Riemann, N. V. Abrosimov, N. Noetzel, M. L. W. Thewalt, and J. J. L. Morton, Phys. Rev. Lett. 105, 067601 (2010).

[5] F. A. Trumbore, Bell Syst. Tech. J. 39, 205 (1960). 
[6] K. Sakamoto, K. Kyoya, K. Miki, H. Matsuhata, and T. Sakamoto, Jpn. J. Appl. Phys. 32, L204 (1993).

[7] E. Abramof, A. F. d. Silva, B. E. Sernelius, J. P. d. Souza, and H. Boudinov, Phys. Rev. B 55, 9584 (1997).

[8] P. Studer, V. Brázdová, S. R. Schofield, D. R. Bowler, C. F. Hirjibehedin, and N. J. Curson, ACS Nano 6, 10456 (2012).

[9] K. Murata, P. L. Neumann, T. Koyano, Y. Yasutake, K. Nittoh, K. Sakamoto, S. Fukatsu, and K. Miki, Jpn. J. Appl. Phys 51, 11PE05 (2012).

[10] K. Miki, J. H. G. Owen, D. R. Bowler, G. A. D. Briggs, and K. Sakamoto, Surf. Sci. 421, 397 (1999).

[11] K. Murata, Y. Yasutake, K. Nittoh, K. Sakamoto, S. Fukatsu, and K. Miki, Appl. Phys. Express 3, 061302 (2010).

[12] J. H. G. Owen, K. Miki, and D. R. Bowler, J. Mater. Sci. 41, 4568 (2006).

[13] J. H. G. Owen, K. Miki, H. Koh, H. W. Yeom, and D. R. Bowler, Phys. Rev. Lett. 88, 226104 (2002).

[14] J. H. G. Owen, F. Bianco, S. A. Köster, D. Mazur, D. R. Bowler, and C. Renner, Appl. Phys. Lett. 97, 093102 (2010).

[15] K. Miki, H. Matsuhata, K. Sakamoto, G. A. D. Briggs, J. H. G. Owen, and D. R. Bowler, Inst. Phys. Conf. Ser. 164, 167 (1999).

[16] Z. Zhong, L. D. Chapman, B. A. Bunker, G. B. Bunker, R. Fischetti, and C. U. Segre, Journal of Synchrotron Radiation 6, 212 (1999).

[17] S. Yagi, W. Yashiro, K. Sakamoto, and K. Miki, Surf. Sci. 595, L311 (2005).

[18] B. Ravel and M. Newville, Journal of Synchrotron Radiation 12, 537 (2005).

[19] A. L. Ankudinov, B. Ravel, J. J. Rehr, and S. D. Conradson, Phys. Rev. B 58, 7565 (1998).

[20] P. Le Fèvre, H. Magnan, and D. Chandesris, Phys. Rev. B 54, 2830 (1996).

[21] P. Hohenberg and W. Kohn, Phys. Rev. 136, B864 (1964).

[22] W. Kohn and L. J. Sham, Phys. Rev. 140, A1133 (1965).

[23] G. Kresse and J. Hafner, Phys. Rev. B 48, 13115 (1993).

[24] G. Kresse and J. Furthmüller, Phys. Rev. B 54, 11169 (1996).

[25] J. P. Perdew, J. A. Chevary, S. H. Vosko, K. A. Jackson, M. R. Pederson, D. J. Singh, and C. Fiolhais, Phys. Rev. B 46, 6671 (1992). 
[26] J. Perdew, K. Burke, and M. Ernzerhof, Phys. Rev. Lett. 77, 3865 (1996).

[27] P. E. Blöchl, Phys. Rev. B 50, 17953 (1994).

[28] G. Kresse and D. Joubert, Phys. Rev. B 59, 1758 (1999).

[29] R. R. Wixom and A. F. Wright, Phys. Rev. B 74 (2006).

[30] H. Seong and L. Lewis, Phys. Rev. B 53, 9791 (1996).

[31] M. Shimomura, K. Miki, T. Abukawa, and S. Kono, Surf. Sci. 447, 6 (2000).

[32] Y. Chen, F. J. García de Abajo, A. Chassé, R. X. Ynzunza, A. P. Kaduwela, M. A. Van Hove, and C. S. Fadley, Phys. Rev. B 58, 13121 (1998).

[33] H. Katayama-Yoshida, K. Kusakabe, H. Kizaki, and A. Nakanishi, Appl. Phys. Express 1, $081703(2008)$

[34] T. Fukushima, H. Katayama-Yoshida, H. Uede, Y. Takawashi, A. Nakanishi, and K. Sato, J. Phys. Condens. Matter 26, 355502 (2014).

[35] K. Iwaya, D. R. Bowler, V. Brázdová, A. F. d. Silva, C. Renner, W. Wu, A. J. Fisher, A. M. Stoneham, and G. Aeppli, Phys. Rev. B 88, 035440 (2013).

[36] A. Saito, K. Matoba, T. Kurata, J. Maruyama, Y. Kuwahara, K. Miki, and M. Aono, Jpn. J. Appl. Phys. 42, 2408 (2003). 